\title{
Overexpression of KIAA0101 Promotes the Progression of Non-small Cell Lung Cancer
}

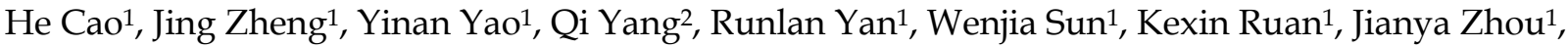 \\ Jianying Zhou ${ }^{\boxplus}$ \\ 1. Department of Respiratory Disease, Thoracic Disease Center, The First Affiliated Hospital, College of Medicine, Zhejiang University, Hangzhou 310003, \\ China \\ 2. Department of Respiratory Disease, The First Affiliated Hospital of Jiaxing, Jiaxing University, Jiaxing 314000, China
}

$\square$ Corresponding author: Jianya Zhou (zhoujy@zju.edu.cn) or Jianying Zhou (zjyhz@zju.edu.cn), Department of Respiratory Disease, Thoracic Disease Center, The First Affiliated Hospital, College of Medicine, Zhejiang University, No.79, Qingchun Road, Shangcheng District, Hangzhou, Zhejiang 310003, China. Tel: +86-0571-87236876; Fax: +86-0571-87236876

(1) The author(s). This is an open access article distributed under the terms of the Creative Commons Attribution License (https://creativecommons.org/licenses/by/4.0/). See http://ivyspring.com/terms for full terms and conditions.

Received: 2020.03.13; Accepted: 2020.09.11; Published: 2020.09.23

\begin{abstract}
Lung cancer is the leading cause of cancer related death worldwide, with a continue-rising incidence. The proliferating cell nuclear antigen binding protein KIAA0101 is highly expressed in various types of cancer, including non-small cell lung cancer (NSCLC). However, its biological role and underlying mechanisms in NSCLC remains unclear. We downloaded KIAA0101 mRNA and clinical data from The Cancer Genome Atlas (TCGA) and Gene Expression Omnibus (GEO), and verified the KIAA0101 expression by conducting experiments of immunochemistry (IHC), immunofluorescence (IF), quantitative real-time PCR (qRT-PCR) and western-blot. Functional experiments were performed to explore the biological roles in vitro and in vivo. The results showed that KIAA0101 was overexpressed in NSCLC tissues and cell lines. High KIAA0101 expression was associated with high T stage, nodal invasion, advanced tumor stage, and poor overall survival $(P<0.01)$. The receiver operating characteristic $(R O C)$ curves showed that KIAA0101 could distinguish NSCLC from paired normal tissues with statistical significance (AUC $=0.969$, $P<0.001)$. The multivariate analysis revealed that KIAA0101 was an independent prognostic factor for overall survival $(\mathrm{HR}=1.249,95 \% \mathrm{Cl}$ : 1.001-1.559, $P=0.049)$. Furthermore, KIAA0101 knockdown induced G1 phase cell cycle arrest and inhibited NSCLC cell proliferation and migration. We also found that the depletion of KIAA0101 decreased tumor volume in nude mice. In summary, our findings suggested that KIAA0101 was a reliable diagnostic and prognostic factor in NSCLC, with potential to be a promising treatment target.
\end{abstract}

Key words: KIAA0101, non-small cell lung cancer, diagnosis, prognosis, proliferation

\section{Introduction}

Lung cancer ranks as the most common cancer worldwide, with lethal outcomes for the metastasis cases. According to GLOBOCAN 2018, the estimated new lung cancer-related death will reach 1.8 million, accounting for $18.4 \%$ of the total cancer-related death [1]. In the absence of obvious symptoms in the early phase, the majority of patients with lung cancer are diagnosed in the advanced stage. Conventional treatments, such as pneumonectomy, lobectomy, radiotherapy, and chemotherapy, show limited efficacy and remain unsatisfactory for patients with metastatic non-small cell lung cancer (NSCLC). In the recent decade, the introduction of immunotherapy into clinical practice has transformed the treatment options with tremendous improvements in the prognosis of patients with tumor disease. However, the death rate of metastasis lung cancer still remains at a relatively high level. Therefore, reliable biomarkers and promising therapeutic targets are urgently needed to inspire novel treatments for lung cancer.

KIAA0101, also known as proliferating cell nuclear antigen (PCNA)-associated factor (PAF15), is a conserved PCNA-binding protein that plays an 
essential role in the regulation of various biological processes [2-4]. KIAA0101 protein competes with the binding of DNA polymerase to regulate DNA replication and damage repair. Recently, KIAA0101 has been reported to play a pro-cancer and anti-cancer role in cancer development. For example, KIAA0101 knockdown significantly induces G1/S phase arrest and inhibits cell growth in breast cancer cells [5]. Furthermore, miR-197-5p targets KIAA0101 to induce cellular senescence in fibrosarcoma [6]. In contrast, KIAA0101 level is decreased in hepatocellular carcinoma (HCC) tissues, and KIAA0101 cDNA transfection can inhibit cell growth in vitro [7]. In summary, the functions of KIAA0101 differs in different types of tumors. A recent study revealed that KIAA0101 expression is upregulated in NSCLC tissue, and patients with high KIAA0101 expression are associated with early postoperative recurrence [8]. However, the biological functional analysis of KIAA0101 in lung cancer cells has never been performed.

Therefore, we aim to investigate whether KIAA0101 expression is associated with clinicopathological characteristics and survival time of patients with NSCLC. Besides, the biological role of KIAA0101 was also explored in vitro and in vivo.

\section{Materials and methods \\ NSCLC tissue samples}

A total of 11 pairs of NSCLC tissues and adjacent normal tissues were collected from Jan. 2018 to Mar. 2018. These patients received surgical lobectomy or pneumonectomy at the First Affiliated Hospital of Zhejiang Medical College, and cases pretreated with radiotherapy and/or chemotherapy were excluded. The study was approved by the Human Research Ethics Committee of the First Affiliated Hospital of Zhejiang Medical College. Written informed consent was provided.

\section{Cell lines}

We purchased HEK293T and NSCLC cell lines (A549, H1299, PC-9, and H226) from American Type Culture Collection (ATCC, Manassas, VA), and obtained MRC-5, a human non-malignant cell line, from the Cell Bank of the Chinese Academy of Medical Sciences (Shanghai, China). H1299 and H226 cells were cultured in RPMI-1640 (Hyclone, Logan, Utah, USA) with $10 \%$ fetal bovine serum (FBS, Gibco, Grand Island, NY, USA), and other cell lines were cultured in Dulbecco's Modified Eagle Medium (DMEM, Hyclone, Logan, Utah, USA) containing 10\% FBS and 1\% penicillin-streptomycin solution. All cells were maintained in an incubator at $37^{\circ} \mathrm{C}$ with $5 \%$ $\mathrm{CO}_{2}$.

\section{RNA isolation and quantitative real-time PCR (qRT-PCR)}

Total RNA was extracted using TRIzol reagent (Invitrogen, Carlsbad, CA), following the manufacturer's protocols. Then we carried out complementary DNA (cDNA) synthesis with a PrimeScript $^{\mathrm{TM}}$ RT reagent Kit (Takara, Dalian, China). Quantitative real-time PCR (qRT-PCR) analysis was performed in a CFX96 real-time PCR Detection System (Bio-Rad, California, USA) using SYBR $^{\circledR}$ Premix Ex Taq ${ }^{\mathrm{TM}}$ II master mix (Takara, Dalian, China). Each test was repeated for three times, and GAPDH was used as an internal control. Relative mRNA expression was calculated using the $2^{-\Delta \Delta \mathrm{Ct}}$ method. All sequences of the primers are listed in Table 1.

Table 1. Sequences of primers and shRNA used in this study.

\begin{tabular}{lll}
\hline \multicolumn{3}{l}{ Primers used for quantitative real-time PCR } \\
\hline Gene & Forward Primer & Reverse Primer \\
KIAA0 & CTCTGCCACTAATTCGACAT & TTCAGAATCTTAGGGGACAAC \\
101 & CA & \\
Cyclin & GCTGCGAAGTGGAAACCAT & CCTCCTTCTGCACACATTTGAA \\
D1 & C & \\
Cyclin & AATAAGGCGAAGATCAACA & TTTGTTACCAATGTCCCCAAGAG \\
B1 & TGGC & \\
CDK2 & CCAGGAGTTACTTCTATGCC & TTCATCCAGGGGAGGTACAAC \\
& TGA & \\
CDK6 & GCTGACCAGCAGTACGAAT & GCACACATCAAACAACCTGACC \\
& G & \\
GADP & TGACTTCAACAGCGACACCC & CACCCTGTTGCTGTAGCCAAA \\
H & A & \\
shRNA & & \\
oligos & & \\
KIAA0 & CCGGGCAACCTGATCACACAAATGACTCGAGTCATTTGTGTGATC \\
101 & AGGTTGCTTTTTG & \\
Control & TTCTCCGAACGTGTCACGTCTCGAGACGTGACACGTTCGGAGAAT \\
& TTTTT \\
\hline
\end{tabular}

\section{Western Blot}

Cells were washed by pre-cold PBS twice, followed by lysis with cold RIPA (Beyotime, Shanghai, China) containing phosphatase inhibitors and protease inhibitors. Protein concentration was calculated using the BCA method. Total proteins were electrophoresed by 12\% SDS-PAGE gel and were transferred onto polyvinylidene fluoride (PVDF, Millipore, Bedford, MA, USA) membranes. Next, the membranes were blocked in $5 \%$ skimmed milk powder and were incubated overnight at $4{ }^{\circ} \mathrm{C}$ with the primary antibody against KIAA0101 (1:1000, Abnova), cyclin D1 (1:1000, Cell Signaling Technology), cyclin B1 (1:1000, Cell Signaling Technology), CDK2 (1:1000, Cell Signaling Technology), CDK6 (1:1000, Proteintech) and $\alpha$-tubulin (1:5000, Proteintech). After that, membranes were incubated with an HRP-conjugated second antibody (1:20000, Beyotime, Shanghai, China) for $1 \mathrm{~h}$ at room temperature. After a wash with $0.1 \%$ 
TBST for three times, target proteins were visualized using an enhanced chemiluminescence system (Bio-Rad, California, USA).

\section{Plasmid construction and cell transfection}

The pLKO.1 plasmid vector (Sigma Aldrich, St Louis, MO, USA) was used to knock down KIAA0101 expression. The targeted sequence and scramble shRNA sequence were synthesized (Tsingke, Hangzhou, China) and then were subcloned in pLKO.1 vector. The detailed shRNA sequences are listed in Table 1. A total of $5 \times 10^{5}$ cells were seeded into six-well plate overnight, and were transfected with $2 \mu \mathrm{g}$ plasmid using Lipofectamin ${ }^{\circledR} 3000$ reagents (Invitrogen, Carlsbad, CA) according to manufacturer's instructions. At $72 \mathrm{~h}$ after transfection, we performed western blot to determine the inhibition efficiency.

\section{Lentivirus package and stable cell culture}

For experiments in vivo, we also constructed stable-transfected cells. Lentivirus was packaged in

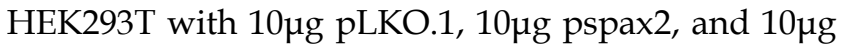
pMD2G plasmids. The cell suspension was centrifuged at 3000rpm to collect virus suspension. Then we mixed PEG8000 with the suspension to purify the virus. To construct a stable-transfected cell line, we infected cells with 200 $\mu$ lentivirus and selected with $2 \mu \mathrm{g} / \mathrm{mL}$ puromycin (GeneChem, Shanghai, China).

\section{Immunohistochemistry}

Four to five micrometer thick formalin-fixed paraffin-embedded (FFPE) sections were deparaffinized and rehydrated in graded solution. After antigen retrieval, sections were incubated overnight at $4^{\circ} \mathrm{C}$ with the KIAA0101 primary antibody (1:200, Abnova, Taiwan, China). After a wash with PBS for three times, goat anti-mouse IgG was incubated for $1 \mathrm{~h}$ at $37^{\circ} \mathrm{C}$. Then, DAB (OriGene, Beijing, China) staining was performed to visualize peroxidase activity. Images were observed with a light microscope (Olympus Corporation, Tokyo, Japan, magnification $\times 400$ ).

\section{Immunofluorescence preparations}

Seeded cells were fixed with $4 \%$ paraformaldehyde for $30 \mathrm{~min}$. After washing for three times with PBS, $0.25 \%$ Triton X-100 was added for permeabilizing membrane. Then cells were blocked with 3\% BSA for $1 \mathrm{~h}$, and were incubated with the KIAA0101 antibody (1:200, Abnova, Taiwan, China) at $4{ }^{\circ} \mathrm{C}$ overnight. On the next day, cells were incubated with Alexa Fluor 594 goat anti-mouse antibody (KeyGen, Nanjing, China). DAPI dilution
(Invitrogen, NY, USA) was incubated to visualize the nuclei. Images were observed with a light microscope (Olympus Corporation, Tokyo, Japan, magnification× 400).

\section{CCK-8 and cell colony formation assay}

The CCK-8 assay was used to assess cell proliferation ability (Dojindo, Kumamoto, Japan). A density of 1000 cells were seeded in 96-well cell plate after $48 \mathrm{~h}$ transfection. In order to test the proliferation

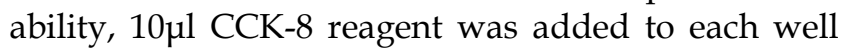
and cells were cultured at $37^{\circ} \mathrm{C}$ incubator for 1 hour. The optical density (OD) values of $450 \mathrm{~nm}$ were measured at $24 \mathrm{~h}, 48 \mathrm{~h}, 72 \mathrm{~h}$, and $96 \mathrm{~h}$, respectively. For cell colony formation, single-cell suspensions were seeded into six-well plates (1000 cells/well). After 10-14 days of cell culture, cells were fixed with $4 \%$ paraformaldehyde and stained with crystal violet solution.

\section{Transwell migration and wound healing assay}

For the transwell migration assay, approximately $1 \times 10^{4}$ cells were seeded into the upper chambers with serum-free DMEM. The lower chambers were filled with 700 $\mu 1$ DMEM containing $10 \%$ FBS. After incubation for $24 \mathrm{~h}$, we removed the cells in the upper side and fixed the cells in the bottom chambers, then stained them with crystal violet. Stained migration cells selected from 3 random fields were pictured and counted. A wound-healing assay was also used to test the migration ability. Cells were scratched using $10 \mu \mathrm{l}$ sterile tips when they reached 90\% confluence. After a wash with PBS twice, the culture medium was replaced with serum-free DMEM. Photos were recorded every $12 \mathrm{~h}$ with a microscope (Olympus Corporation, Tokyo, Japan, migration $\times 100)$.

\section{Cell cycle assay}

Cells were harvested after a transfection with shRNA for $48 \mathrm{~h}$ and were fixed with pre-cooled ethanol for over $4 \mathrm{~h}$. After being washed with PBS, cells were stained with propidium iodide/RNase (Yeasen, Shanghai, China) for $15 \mathrm{~min}$ in dark. Then cell cycle analyses were performed by flow cytometry (FACS Verse, BD Biosciences, MA, USA).

\section{Tumor in vivo}

Male nude mice (4-6 weeks old) were purchased from Shanghai SLAC Laboratory Animal Co. Ltd. The mice were randomly divided into two groups, with three mice in each group. After a week adjustment to local conditions, mice were subcutaneously implanted with $1 \times 10^{6}$ PC-9 cells. The sizes of xenograft tumors were recorded every 2-3 days. The tumor volume was 
calculated as $0.5 \times$ length $\times$ width $^{2}$. The Ethics Committee approved all animal studies.

\section{Bioinformatic analysis of public databases}

We downloaded the level 3 data from The Cancer Genome Atlas (TCGA, https://portal.gdc .cancer.gov/). GSE19804 and GSE18842 were also downloaded from Gene Expression Omnibus (GEO) (https://www.ncbi.nlm.nih.gov/geo/). Data in count form was normalized into $\log 2$ form for analysis.

\section{Gene set enrichment analysis (GSEA)}

To identify relevant biological pathways, we performed GSEA to determine a significant and concordant difference that existed between two types of physiological states. A gene set was considered to enrich when the standard $P$-value was $<0.05$ and the false discovery rate (FDR) was $<0.25$.

\section{Statistical analysis}

Statistical analyses were performed with SPSS software 22.0 and GraphPad Prism 7.0 (La Jolla, CA, USA). The differences between categorical variables were compared with a chi-square test. The receiver operating characteristic (ROC) curves and area under the curve (AUC) were used to calculate the diagnostic value in patients with NSCLC. Survival curves were performed using the Kaplan-Meier method with the log-rank test. Next, univariate and multivariate analyses were performed to identify the risk factors. A $P$-value less than 0.05 was defined as statistically significant for all analyses.

\section{Results}

KIAA0101 was significantly overexpressed and associated with clinical parameters in NSCLC.

To find out the expression of KIAA0101 in NSCLC, we downloaded mRNA expression data from TCGA, which included 1037 lung tumor tissues and 108 adjacent normal lung tissues. As shown in Figure 1A-C, KIAA0101 was significantly upregulated in 502 LUSC and 535 LUAD tumor tissues compared with that in normal tissues $(P<0.001)$. Analysis results from GSE19804 and GSE18842 datasets confirmed the overexpression of KIAA0101 in lung cancer tissues (all $P<0.001$, Figure 1D and E). Next, we assessed the correlation between KIAA0101 expression and different clinicopathological parameters. KIAA0101 upregulation was positively correlated with advanced $\mathrm{T}$ stage, nodal invasion, tumor stage, and poor outcome (Figure 1F-I; Table 2). However, no difference was observed between the patients of aged $\geq 60$ and aged $<60$ (Table 2).
Table 2. Comparison of the association between KIAA0101 expression and clinicopathological parameters in patients with NSCLC.

\begin{tabular}{|c|c|c|c|c|}
\hline \multirow[t]{2}{*}{ Characteristics } & \multicolumn{2}{|c|}{ KIAA0101 expression } & \multirow[t]{2}{*}{$\chi^{2}$} & \multirow[t]{2}{*}{$P$ value } \\
\hline & Low $(\mathrm{N}=499)$ & High $(\mathrm{N}=502)$ & & \\
\hline \multicolumn{5}{|l|}{ Age (years) } \\
\hline$<60$ & 111 & 117 & 0.190 & 0.663 \\
\hline$\geq 60$ & 381 & 376 & & \\
\hline \multicolumn{5}{|l|}{ Gender } \\
\hline Female & 250 & 149 & 43.525 & $<0.001^{*}$ \\
\hline Male & 249 & 353 & & \\
\hline \multicolumn{5}{|l|}{ T stage } \\
\hline $\mathrm{T} 1+\mathrm{T} 2$ & 425 & 416 & 1.157 & 0.282 \\
\hline $\mathrm{T} 3+\mathrm{T} 4$ & 72 & 85 & & \\
\hline \multicolumn{5}{|l|}{ N stage } \\
\hline No & 340 & 303 & 8.784 & $0.003^{*}$ \\
\hline $\mathrm{N} 1+\mathrm{N} 2+\mathrm{N} 3$ & 146 & 194 & & \\
\hline \multicolumn{5}{|l|}{ M stage } \\
\hline M0 & 342 & 402 & 0.632 & 0.427 \\
\hline M1 & 17 & 15 & & \\
\hline \multicolumn{5}{|l|}{ TNM stage } \\
\hline I-II & 398 & 394 & 0.406 & 0.524 \\
\hline III-IV & 94 & 103 & & \\
\hline \multicolumn{5}{|l|}{ Status } \\
\hline Alive & 353 & 316 & 6.857 & $0.009^{*}$ \\
\hline Dead & 146 & 186 & & \\
\hline
\end{tabular}

* $P<0.05$; NSCLC, non-small cell lung cancer; TNM stage, tumor-node-metastasis stage.

\section{The prognostic value of high KIAA0101 expression in NSCLC}

A Kaplan-Meier survival curves was generated with a log-rank test to explore the association between KIAA0101 expression and overall survival in patients with NSCLC. Figure 2A showed that patients with high KIAA0101 expression exhibited a significantly inferior overall survival compared with those in the low expression group $(P=0.043)$. Figure 2B-E indicated that KIAA0101 expression was a powerful predictive tool for prognosis in patients with metastatic NSCLC $(P=0.010)$, aged $\geq 60 \quad(P=0.026)$, T3+T4 stage $(P=0.009)$, and without nodal invasion NSCLC $(P=0.016)$. In addition, Cox regression analysis confirmed that KIAA0101 expression was an independent risk factor for overall survival (HR=1.249, 95\% CI: 1.001-1.559, $P=0.049$; Table 3).

Table 3. Univariate and multivariate analysis of OS in patients with NSCLC.

\begin{tabular}{|c|c|c|c|c|c|c|}
\hline \multirow[t]{2}{*}{ Characteristics } & \multicolumn{3}{|c|}{ Univariate analysis } & \multicolumn{3}{|c|}{ Multivariate analysis } \\
\hline & $\begin{array}{l}P \\
\text { value }\end{array}$ & HR & $95 \% \mathrm{CI}$ & $P$ value & HR & 95\%CI \\
\hline Age $(\geq 60 /<60$ years) & 0.137 & 1.231 & $0.936-1.619$ & & & \\
\hline Gender (Male/Female) & 0.078 & 1.225 & $0.977-1.537$ & & & \\
\hline TNM stage (III-IV/I-II) & $<0.001^{*}$ & 2.081 & $1.639-2.642$ & & & \\
\hline $\mathrm{N}$ stage $(\mathrm{N} 1+\mathrm{N} 2+\mathrm{N} 3 / \mathrm{N} 0)$ & $<0.001^{*}$ & 1.708 & $1.372-2.125$ & $0.007^{\star}$ & 1.426 & $1.102-1.845$ \\
\hline $\mathrm{T}$ stage $(\mathrm{T} 1+\mathrm{T} 2 / \mathrm{T} 3+\mathrm{T} 4)$ & $<0.001^{*}$ & 1.929 & $1.480-2.514$ & $0.004^{*}$ & 1.581 & $1.155-2.166$ \\
\hline M stage (M1/M0) & $0.004^{*}$ & 2.107 & $1.268-3.499$ & & & \\
\hline $\begin{array}{l}\text { KIAA0101 expression } \\
\text { (High/Low) }\end{array}$ & $0.044^{*}$ & 1.250 & $1.006-1.553$ & $0.049^{*}$ & 1.249 & $1.001-1.559$ \\
\hline
\end{tabular}




\section{The diagnostic value of high KIAA0101 expression in NSCLC}

ROC curves were used to investigate the diagnostic role of KIAA0101. Results showed that KIAA0101 could sufficiently distinguish NSCLC from paired normal tissues (Figure 3A, AUC $=0.969$, $P<0.001)$. We performed ROC analysis in other subgroups and found that high KIAA0101 expression may be a potential prognostic indicator for patients with following characteristic: Male vs. Female (Figure $3 \mathrm{~B}, \mathrm{AUC}=0.629, P<0.001$ ); Alive vs. Dead (Figure 3C, AUC $=0.572, \quad P<0.001$ ); N0 vs. N1 (Figure 3D, AUC $=0.570, P<0.001)$.

\section{KIAA0101 overexpression was verified in NSCLC cell lines and tissues}

To verify the KIAA0101 expression, qRT-PCR and western blot were performed. The mRNA level of KIAA0101 was significantly up-regulated in several lung adenocarcinoma and lung squamous carcinoma cell lines than in the MRC-5 cells (Figure 4A). Western blotting showed similar results in the protein level (Figure 4B). Besides nuclei location in immunofluorescence, we also observed a similar protein expression profile consistent with the western-blot method (Figure 4C). The expression of KIAA0101 was also detected in 11 pairs of NSCLC tissues, KIAA0101 protein mainly located near the nucleus and was overexpressed in eight out of 11 cancer tissues (Figure 4D).
A

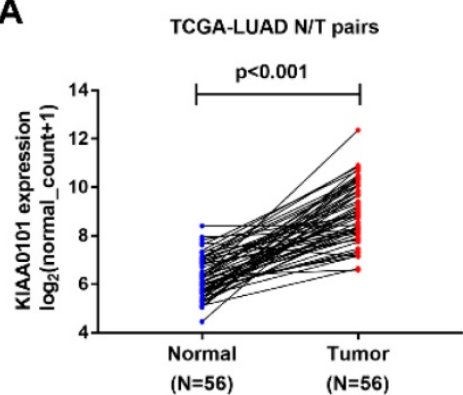

D

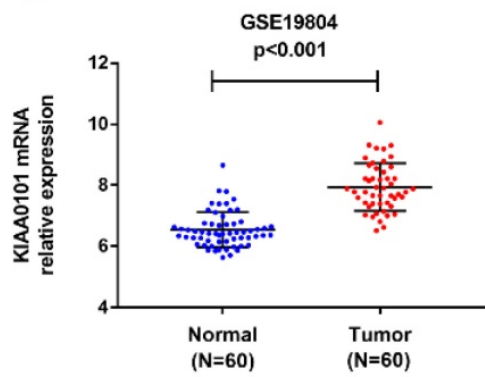

G

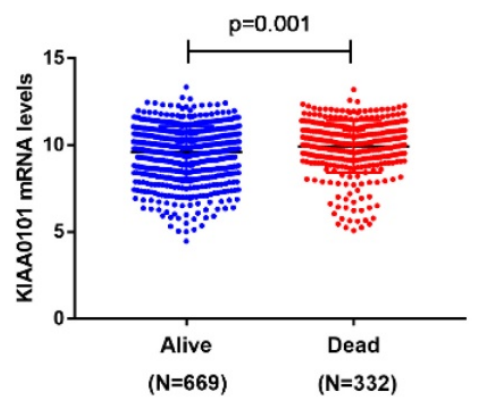

B

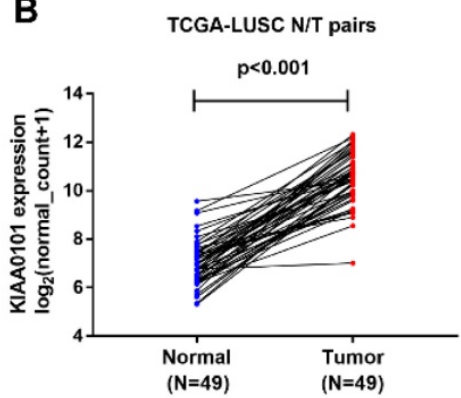

E

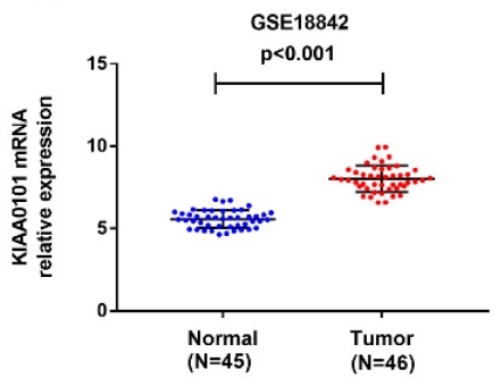

H

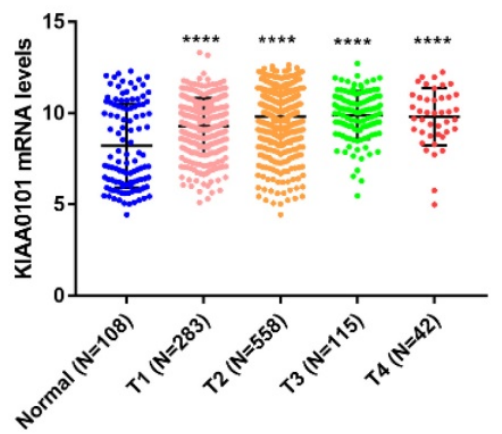

C

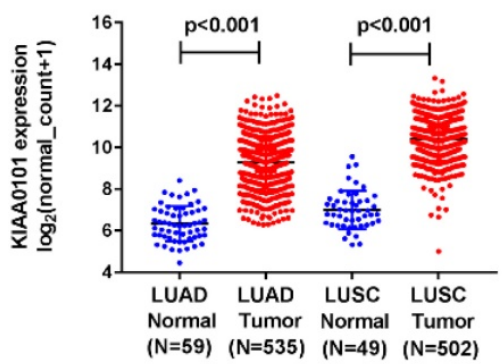

F

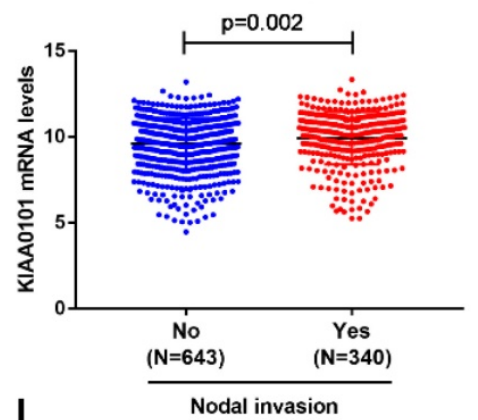

I

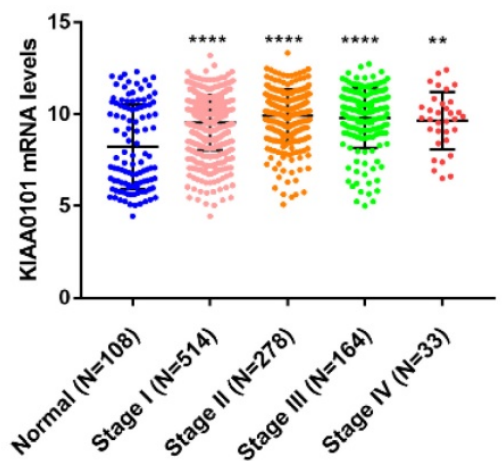

Figure 1. KIAA0101 expression was significantly overexpressed and related to various clinical parameters in NSCLC patients. KIAA0101 expression was upregulated in 56 LUAD (A) and 49 LUSC (B) tissues than paired normal tissues. KIAA0101 mRNA overexpressed in NSCLC tissues in (C) TCGA, (D) Lu lung dataset, (E) Ramos lung dataset. High KIAA0101 expression positively related to various clinical parameters: $(F)$ nodal invasion, $(\mathrm{G})$ poor outcome, $(\mathrm{H})$ advanced $\mathrm{T}$ stage, and $(\mathrm{I})$ tumor stage. $* *, P<0.01$; $* * * *, P<0.0001$. 
A

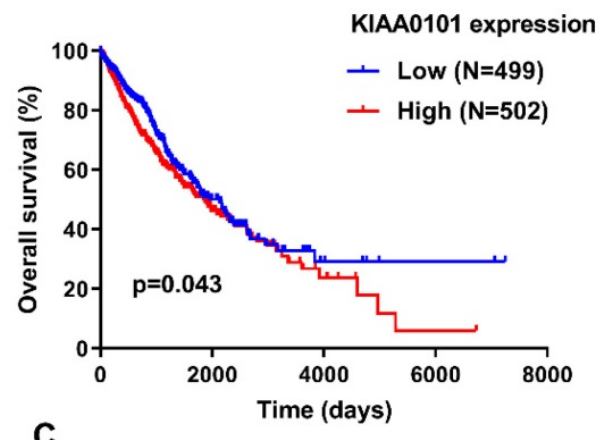

C

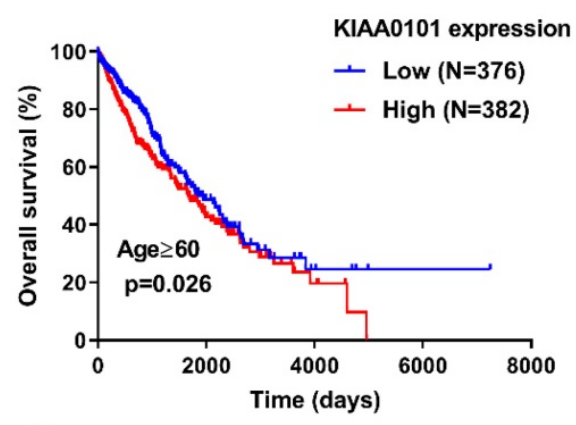

E

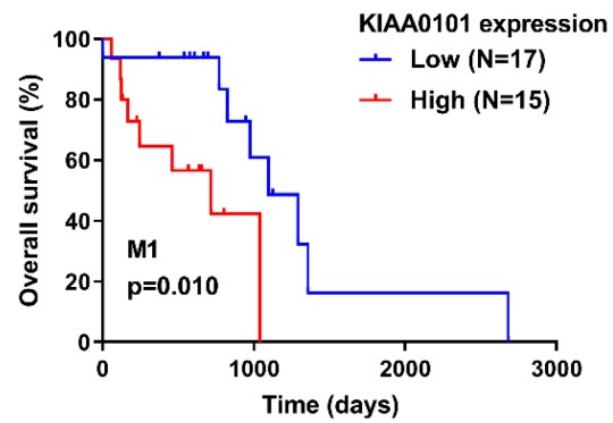

B
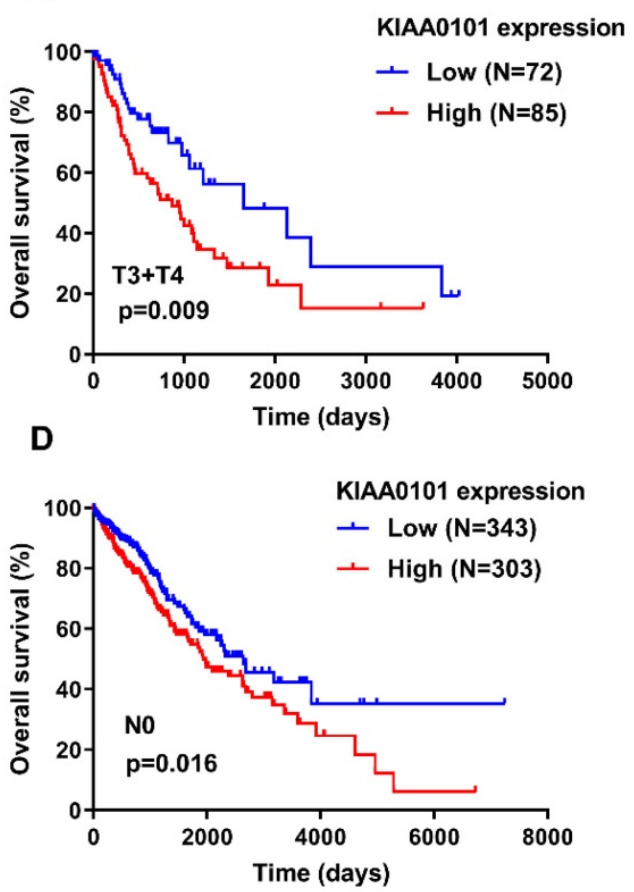

Figure 2. High KIAA0101 expression was associated with a significantly inferior overall survival in NSCLC patients. (A) High KIAA0101 expression group exhibited a significantly inferior overall survival compared with those in the low expression group. Survival analyses were conducted in the subgroups of patients with NSCLC: (B) T3+T4 stage, (C) aged $\geq 60$, (D) without nodal invasion, and (E) metastasis.

\section{The knockdown of KIAA0101 expression inhibited NSCLC proliferation in vitro and in vivo.}

The shRNA oligos were utilized to silence KIAA0101 expression. After transfection for 48h, mRNA levels of KIAA0101 were significantly decreased compared with the negative control group in A549 and PC-9 cells ( $P<0.001$, Figure 5A). Western blot assays showed significant depletions in KIAA0101 protein at 72h (Figure 5B). In the results of CCK-8 assays (Figure 5C), optical density (OD) values in the sh-KIAA0101 group decreased significantly at $24 \mathrm{~h}, 48 \mathrm{~h}, 72 \mathrm{~h}$, and 96h $(P<0.001)$, respectively. For colony formation assay (Figure 5D), negative control group visualized more colonies after 10-14 days' culture in A549 (285.7 \pm 18.8 vs. $137.0 \pm 8.7 ; P=0.002)$ and PC-9 cells $(142.3 \pm 24.3$ vs. $61.3 \pm 12.9 ; P=0.042)$. In the xenograft model, we observed that the sh-KIAA0101 group showed remarkable decrease in tumor volume compared to the control group (Figure 5E).

\section{The knockdown of KIAA0101 expression inhibited NSCLC migration in vitro.}

To evaluate the effect of KIAA0101 on the ability of migration, wound-healing and transwell assay were used. As shown in Figure 6A, the wound healing rate in the negative control group was $77.8 \pm 2.0 \%$ in A549 cells and $83.9 \pm 5.8 \%$ in PC-9 cells after $24 \mathrm{~h}$ serum-free DMEM incubation. However, the sh-KIAA0101 group only showed a healing rate of $31.5 \pm 4.8 \% \quad(P<0.001)$ and $55.6 \pm 10.6 \% \quad(P=0.02)$. In transwell assay (Figure 6B), migration ability was significantly suppressed after KIAA0101 depletion. The number of migrated A549 and PC-9 cells was $324.3 \pm 13.0$ and $800.0 \pm 7.6$, respectively, and the number of migrated cells sharply decreased to 197.3 \pm 5.2 and 305.7 \pm 9.3 in sh-KIAA0101 group. 


\section{A}

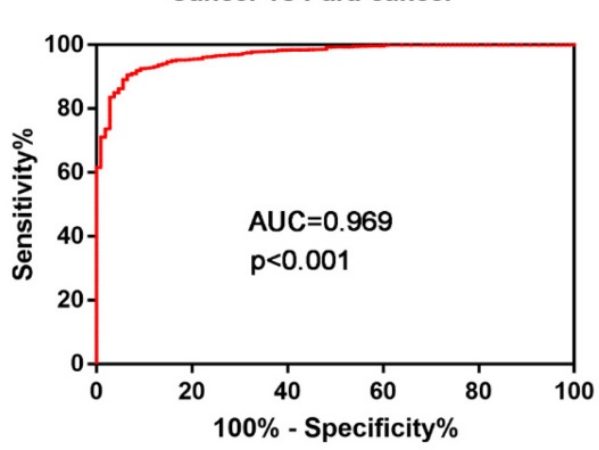

C

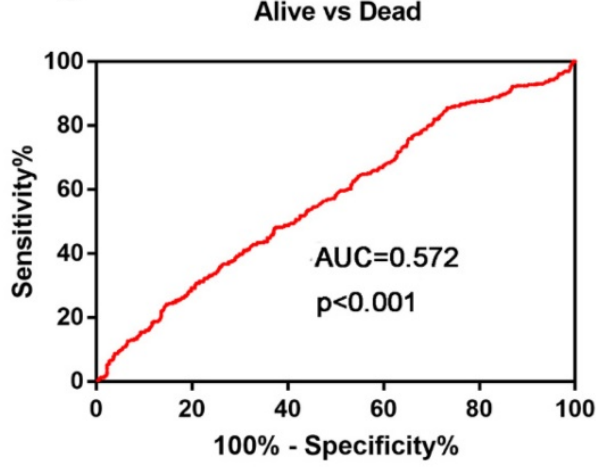

B

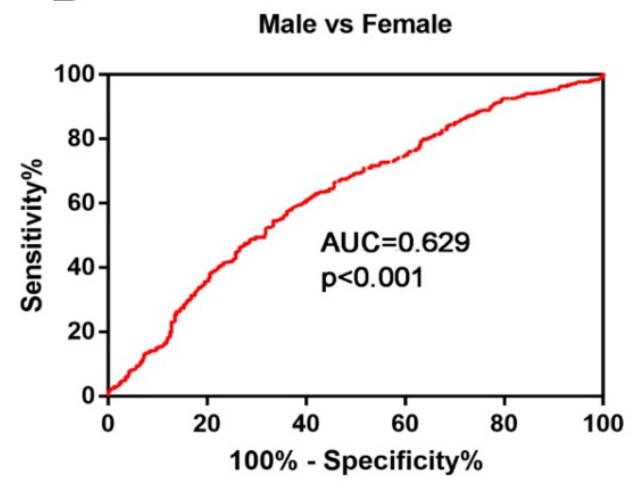

D

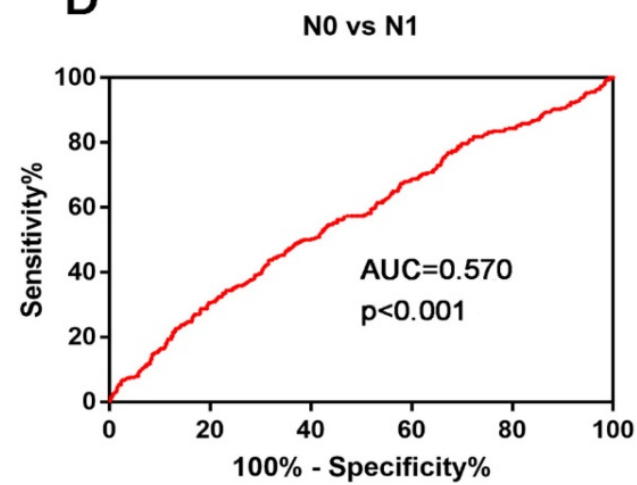

Figure 3. KIAA0101 expression played a potential diagnostic role in NSCLC. (A) KIAA0101 sufficiently distinguish NSCLC from paired normal tissues. ROC subgroup analyses showed that KIAA0101 expression might be a potential prognostic indicator with the following characteristic: (B) Male vs. Female; (C) Alive vs. Dead; (D) NO VS N1.

A

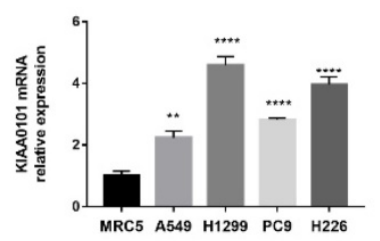

C
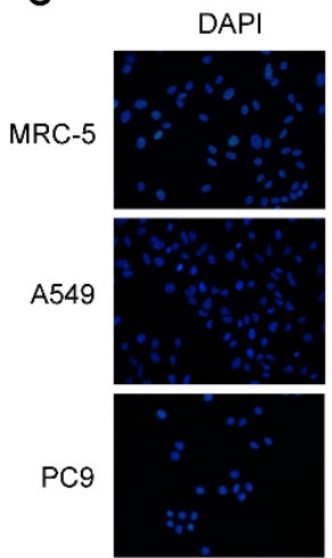

KIAA0101
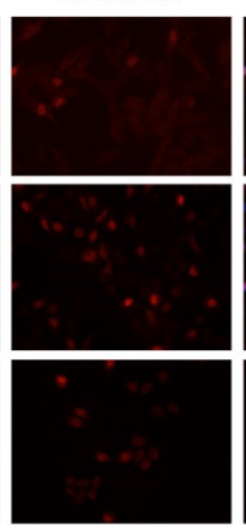

B

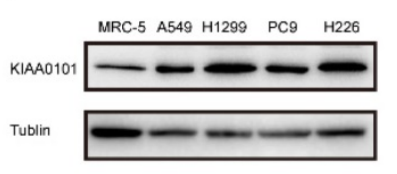

D

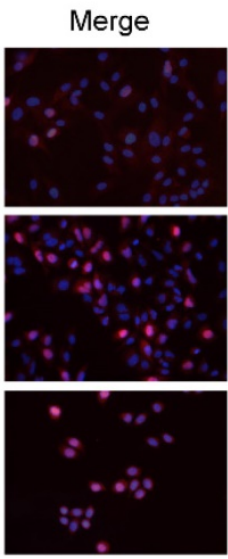

Case 1

Case 2

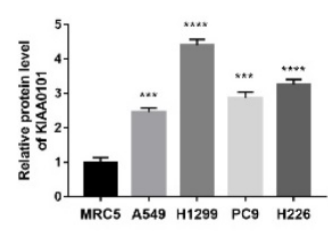

$N(400 x)$
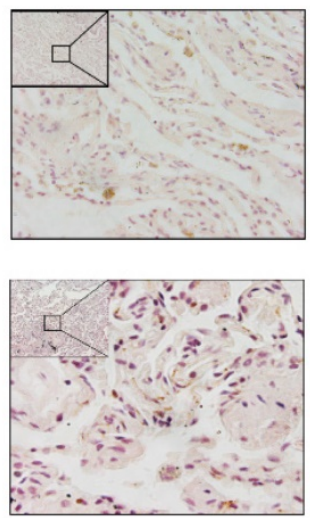

$\mathrm{T}(400 \mathrm{x})$
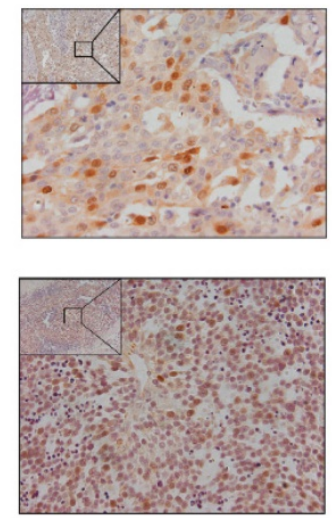

Figure 4. KIAA0101 overexpression was verified in NSCLC cell lines and tissues. (A) qRT-PCR and (B) western-blot results of KIAA0101. (C) Immunofluorescence of KIAA0101 in NSCLC cell lines (magnification $\times 400$ ). (D) Immunochemistry staining of KIAA0101 in NSCLC tissues and adjacent normal tissues (magnification $\times 100$ and $\times 400)$. $* *$, $P<0.01 ; * * *, P<0.001 ; * * * *, P<0.0001$.

\section{KIAA0101 was involved in the TP53 pathway and cell cycle.}

To identify how KIAA0101 influences tumor development, we performed GSEA with TCGA data and used c2.cp.kegg.v6.2.symbols.gmt as the reference gene. Results showed high KIAA0101-related genes were significantly enriched in TP53 signaling pathway (NES=1.95, $P<0.001$, $\mathrm{FDR}=0.078$; Figure 7A) and cell cycle (NES=1.94, 
$P<0.001, F D R=0.047 ;$ Figure $7 \mathrm{~B})$. The influence of KIAA0101 on cell cycle was also investigated by flow cytometry. As shown in Figure 8A-B, KIAA0101 knockdown significantly increased the number of cells in G1 phase compare to the control group $(P<0.05)$. At the molecular level, the expression of cyclin D1, cyclin B1, CDK2, CDK6 were decreased in sh-KIAA0101 treated cells (Figure 8C-D).

\section{Discussion}

NSCLC is a common malignancy with high incidence, as the leading cause of cancer-related mortality. Due to the unobvious early symptoms, over $50 \%$ of the patients with NSCLC are diagnosed in an advanced stage at the first visit. Traditional surgery and chemotherapies remain unsatisfactory for those patients. Immunotherapy has continued to establish indications expanding across several cancers in recent years and preliminary clinical data suggest that the cytotoxic T-lymphocyte-associated antigen 4 (CTLA-4) and programmed death 1 (PD-1) inhibitors are promising approaches for tumor managements [9]. However, the 5-year survival in patients with advanced lung cancer still remains less than $20 \%$. Therefore, novel potential biomarkers and personalized therapeutic targets are urgently needed.

In this study, we found KIAA0101 was overexpressed in NSCLC tissues compare to corresponding normal tissues, and it was useful for diagnosis and prognosis prediction in NSCLC. Besides, the decreased KIAA0101 inhibited cell proliferation and migration abilities, and induced G1 cell cycle arrest in NSCLC cells in vitro and in vivo.
A

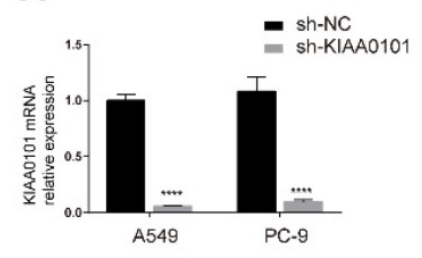

C

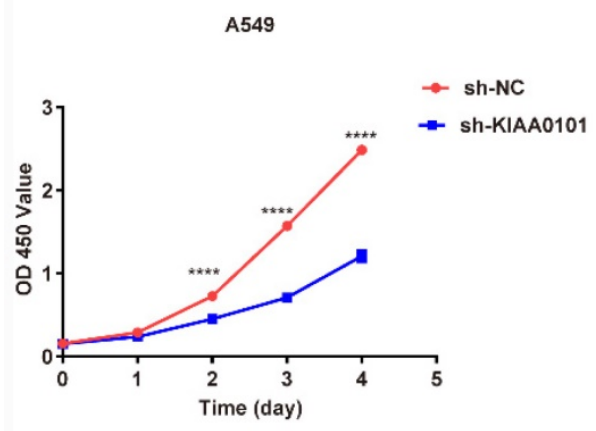

PC-9

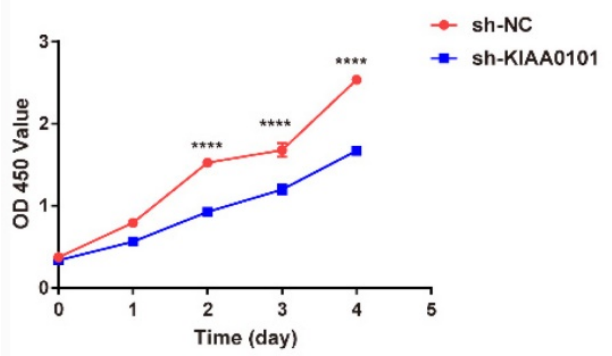

B
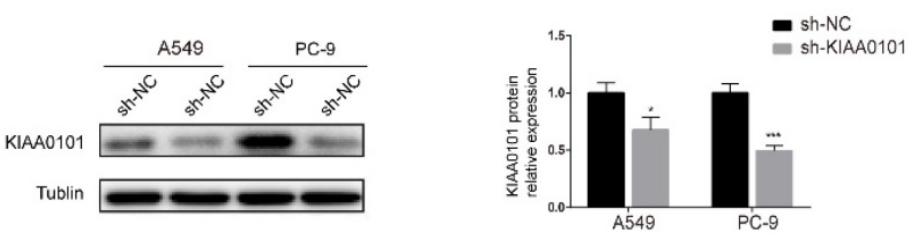

D
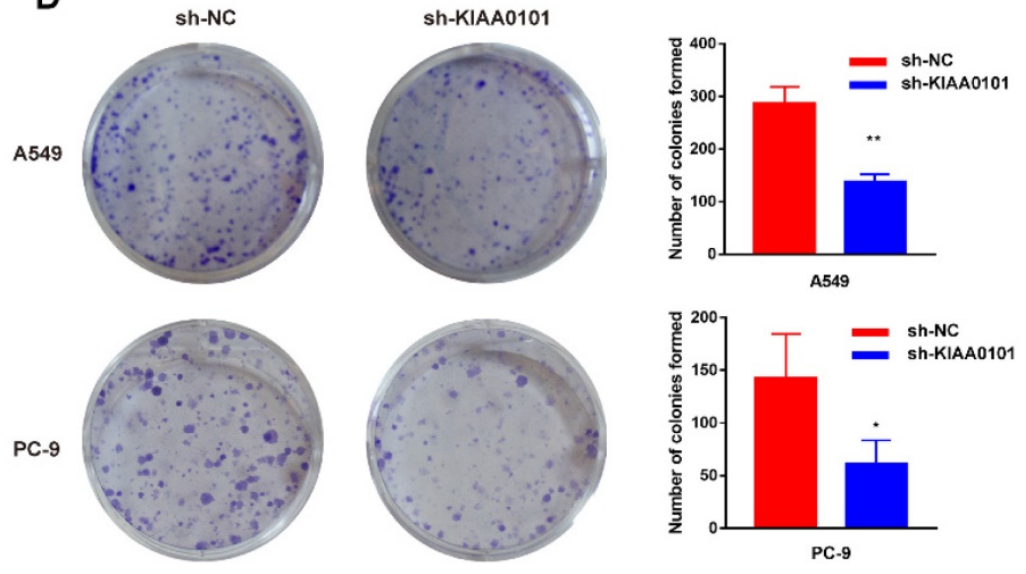

E

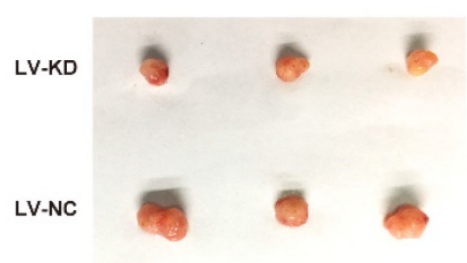

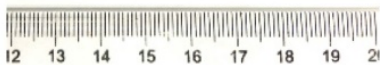

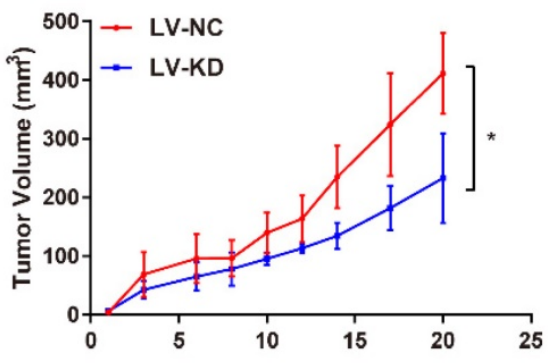

Figure 5. The knockdown of KIAA0101 inhibited NSCLC proliferation in vitro and in vivo. After transfection for $48 \mathrm{~h}-72 \mathrm{~h}$, (A) mRNA and (B) protein levels of KIAA0101 significantly reduced. (C-D) The proliferation capacity of A549 and PC-9 cells decreased according to CCK-8 and colony formation assay. (E) cell growth in the xenograft model. $*, P<0.05 ; * *, P<0.01$;***, $P<0.001 ; * * * *, P<0.0001$. 
A

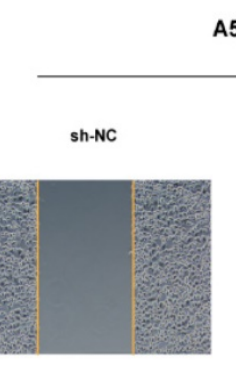

A549

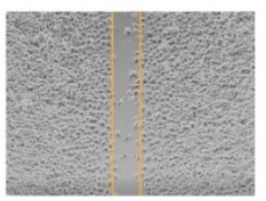

B
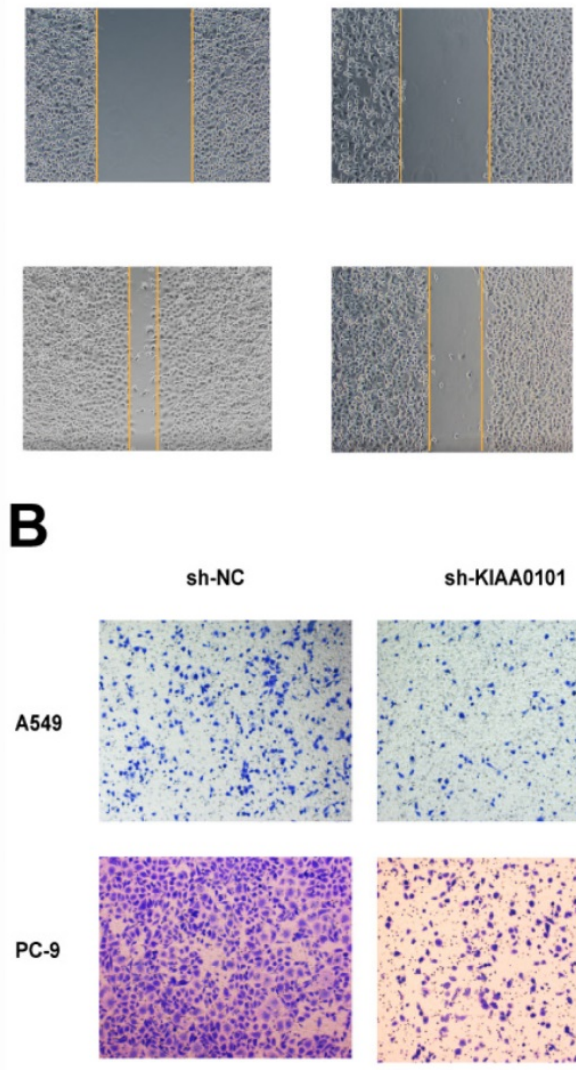

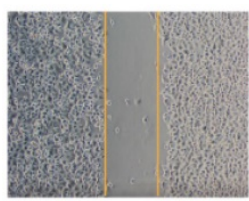

sh-KIAA0101

on

$24 \mathrm{~h}$
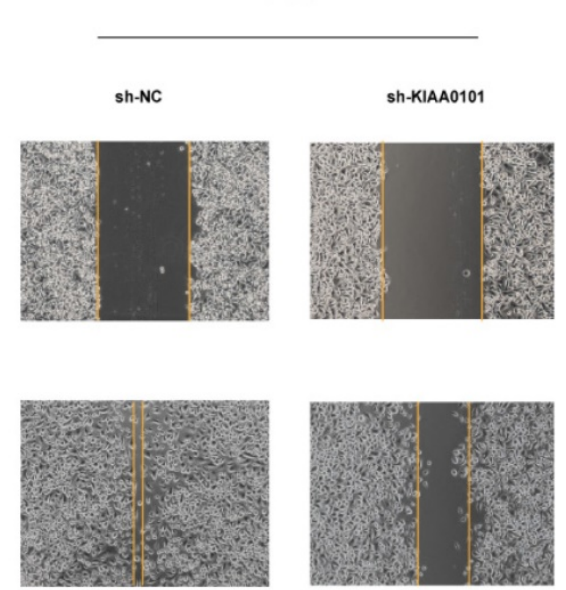

PC-9

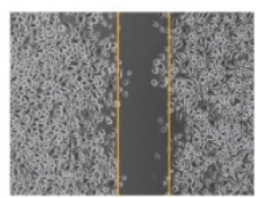

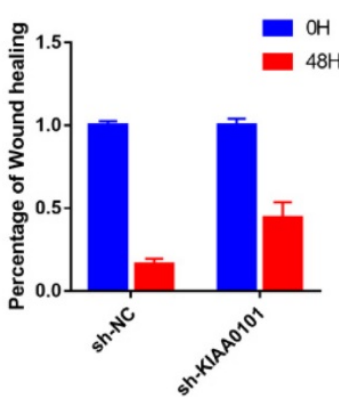

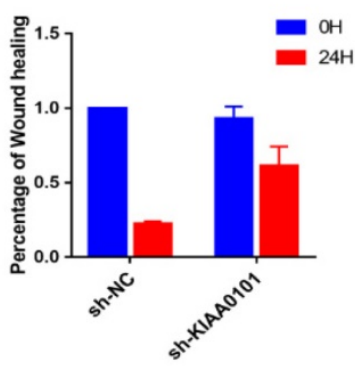

Figure 6. The knockdown of KIAA0101 expression inhibited NSCLC migration in vitro. Representative images of woung-healing using (A) A549 and (B) PC-9 (magnification $\times 100$ ). Representative images of transwell using (C) A549 and (D) PC-9. (magnification $\times 400$ ). $* * *, P<0.001 ; * * * *, P<0.0001$.

\section{A}

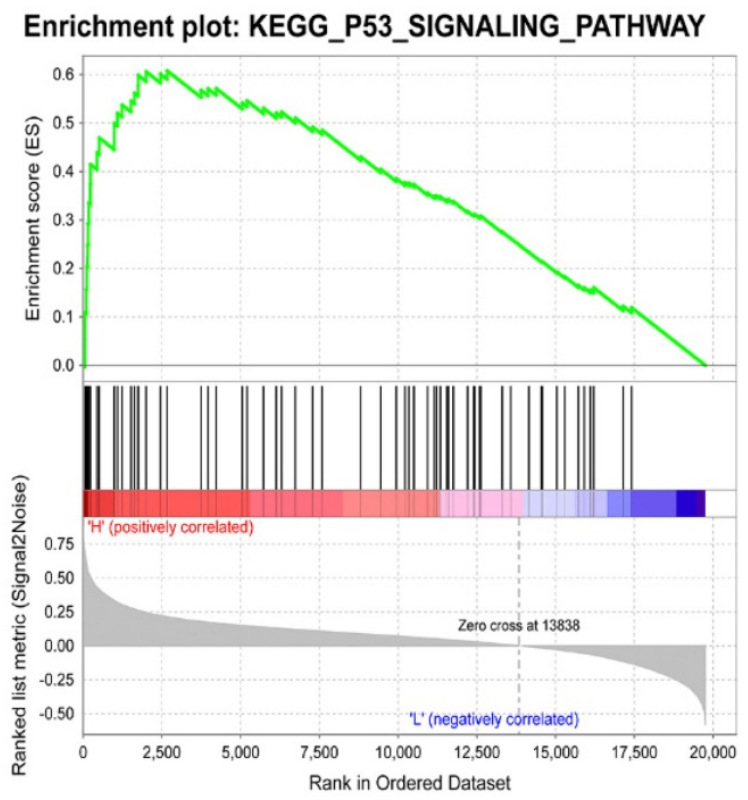

—Enrichment profile - Hits - - Ranking metric scores
B

Enrichment plot: KEGG_CELL_CYCLE

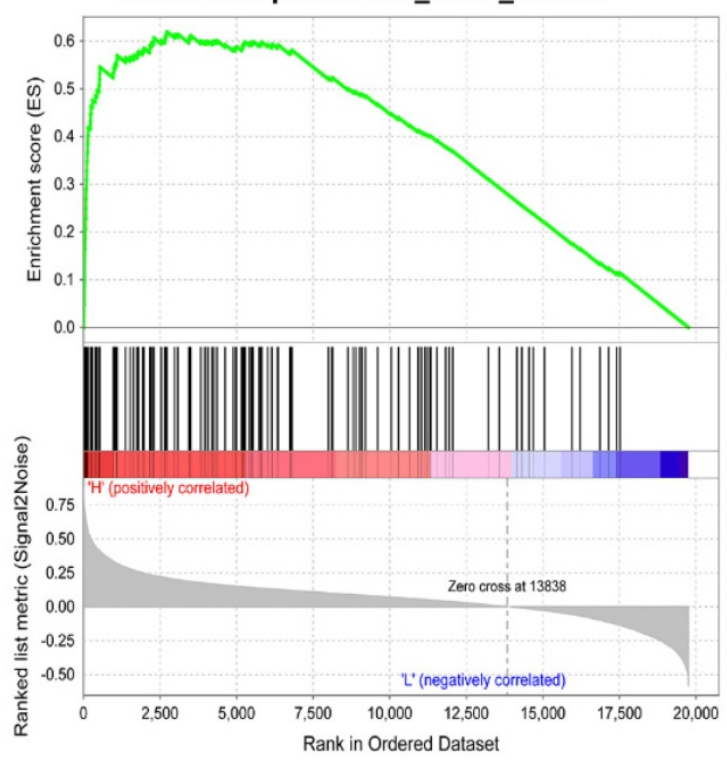

- Enrichment profile - Hits - - Ranking metric scores

Figure 7. Identification of KIAA0101-related pathways with GSEA. High KIAA0101-related genes significantly enriched in (A) TP53 signaling pathway and (B) cell cycle. 
A

A549 sh-NC

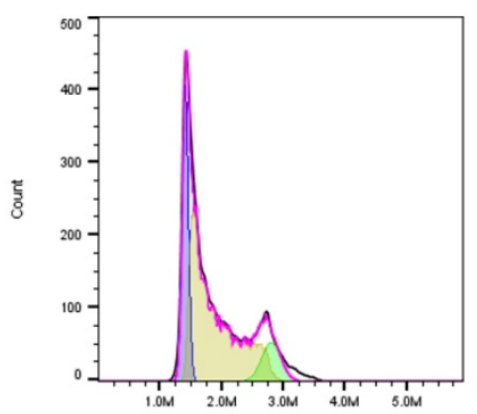

B
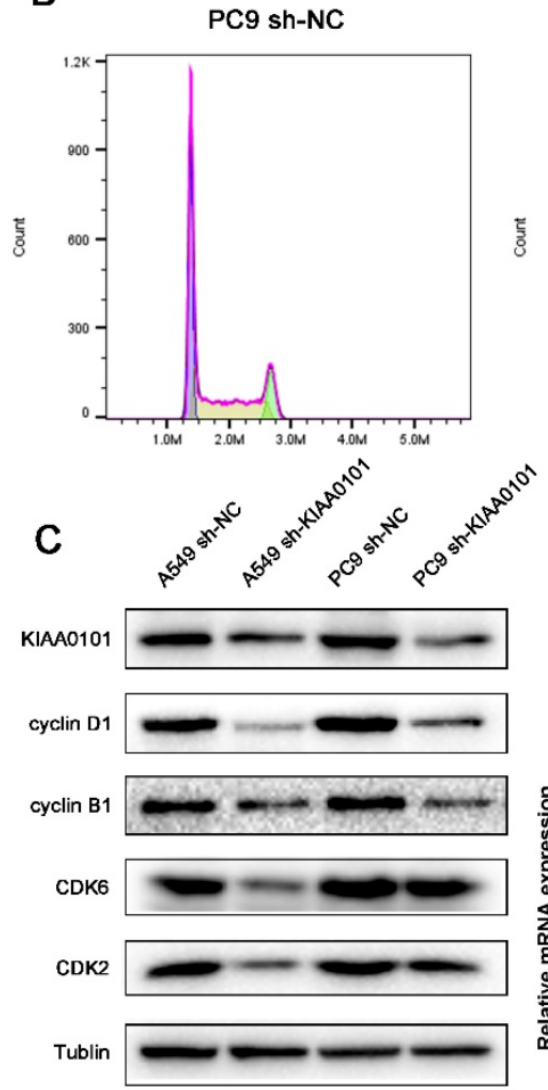
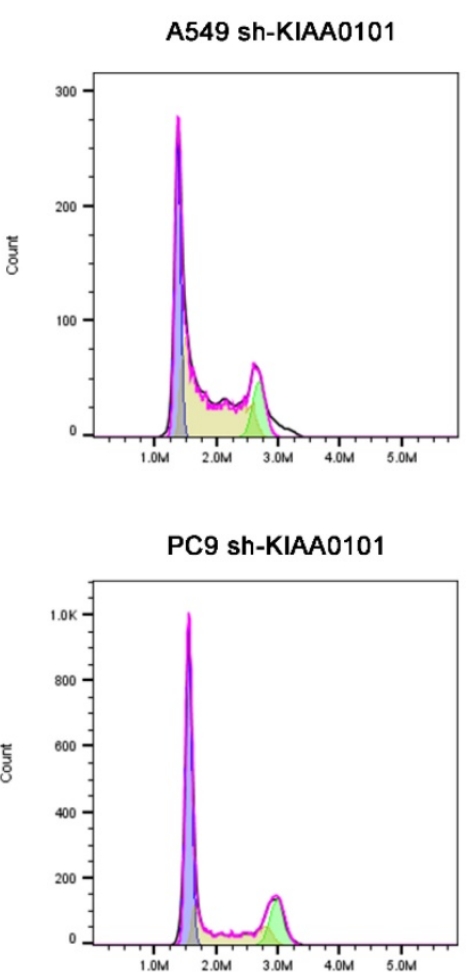

A549

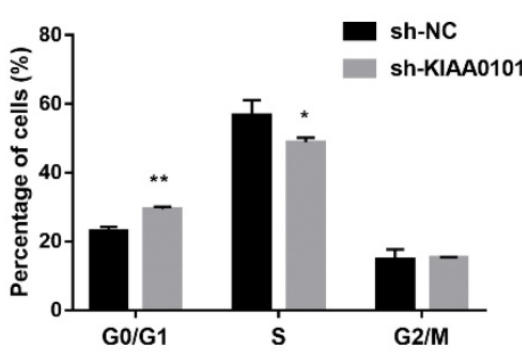

PC9

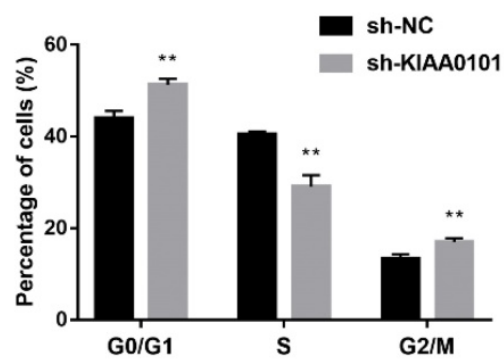

D

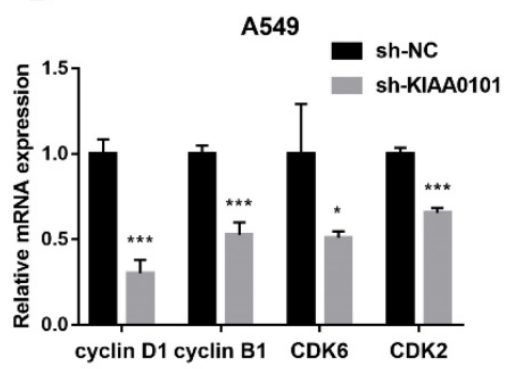

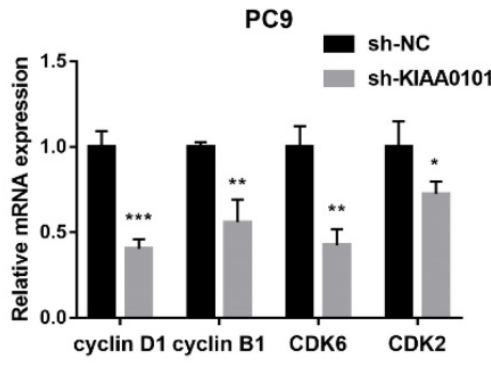

Figure 8. KIAA0101 knockdown induced G1 phase cell cycle arrest. Flow cytomatric analysis of cell cycle distribution of A549 (A) and PC-9 (B) cells. Western blot and qRT-PCR showed decreased expression of cyclin D1, cyclin B1, CDK2, and CDK6 in protein (C) and mRNA level (D). *, $P<0.05 ; * *, P<0.01$.

KIAA0101, also named p15 PAF (PCNA-associated factor) and OEATC-1 (overexpressed in anaplastic thyroid carcinoma-1), was first discovered as a conserved protein that interacted with PCNA $[2,10]$. By competing with PCNA binding, KIAA0101 involves in the regulation processes of DNA replication, DNA repair, and cell cycle $[4,11]$. Recent researches focus on its oncogenic role and indicate that KIAA0101 level is markedly increased in various types of cancer, including breast [5], adrenal [12], ovarian [13], and gastric cancer [14]. In this study, we observed an increased expression of KIAA0101 in NSCLC tissues than in the paired normal tissues as well as in NSCLC cells. TCGA and GEO analysis also confirmed these results. In a previous study conducted by Tatsuya et al. [8], they analyzed the KIAA0101 mRNA expression in 12 NSCLC cases and found that KIAA0101 was overexpressed in eight out of 12 cases, which was consistent with our report.

We discovered that high KIAA0101 expression was positively correlated with nodal invasion, advanced tumor stage, and inferior overall survival, and it was considered as a powerful prognostic indicator for NSCLC. Further analysis showed that KIAA0101 expression and nodal invasion were independent risk factors. Our results were supported by several previous studies with similar results. Abdelgawad et al. revealed that KIAA0101 mRNA in 
the peripheral blood was associated with distant metastasis and advanced stage in patients with hepatocellular carcinoma. Moreover, KIAA0101 mRNA exhibited better sensitivity and specificity than those of alpha-fetoprotein (AFP) and carcinoembryonic antigen (CEA) [15]. In the tumor setting of human gastric cancer, Zhu et al. reported that increased KIAA0101 was a risk marker for recurrence [14]. As for the possible mechanism behind the pro-tumor effect, KIAA0101 was reported to be involved in tumor invasion and recurrence by promoting epithelial-to-mesenchymal transition (EMT) by Zhang et al. and Jin et al. [13,16]. Based on the prior literature, we speculate that KIAA0101 might partly involve in EMT in NSCLC. Notably, this hypothesis is supported by the fact that after reducing KIAA0101 expression, we observed a decreased migration ability in cells.

The proliferation ability was significantly reduced in A549 and PC-9 cells after knockdown of KIAA0101. Moreover, in the xenograft model, the tumor volume significantly decreased compared to the control group. To further reveal the potential mechanism, we performed GSEA and found that KIAA0101 related genes mostly affected TP53 signaling pathway and cell cycle. As a critical tumor suppressor, wild type TP53 protects cells from oncogenesis by maintaining the genome stabilities [17]. TP53 protein activates a panel of genes to repair genome damage when cells are under stress. Also, some studies report that TP53 can interact with some G1 or G2/M checkpoint proteins to induce cell cycle arrest [18]. In our study, flow cytometric analyses confirmed that KIAA0101 was involved in NSCLC cell cycle progression, and the depletion of KIAA0101 induced cells cycle arrest in G1 phase. Moreover, we observed a significant decrease in cyclin D1, cyclin B1, CDK2, and CDK6. These proteins tightly control the cell cycle progression, with critical roles in DNA replication and mitosis. On the other hand, they are also important downstream factors in the TP53 pathway [19]. Lv et al. reported the network of KIAA0101, TP53, SP1 functioned in breast cancer, and the depletion of KIAA0101 suppressed cell cycle progression. In luciferase reporter assay and ChIP assay, they found KIAA0101 disrupted the interaction between TP53 and SP1 [5]. Based on these evidence, we assume that KIAA0101 could influence cell proliferation by TP53 network and cell cycle.

To the best of our knowledge, the present study first reported the oncogenic role of KIAA0101 by integrating bioinformatics with functional cell experiments. However, there are some shortcomings in our study. The mechanism of KIAA0101 overexpression remains unclear, and the molecular mechanism to promote cancer progression has not been fully elucidated. More works are required to be done to clarify these issues.

In conclusion, KIAA0101 is overexpressed in NSCLC with important diagnostic and prognostic roles. Furthermore, KIAA0101 promotes cancer progression by enhancing cell migration and proliferation, which provides a new clue to develop more efficient therapies in the future.

\section{Abbreviations}

PCNA: proliferating cell nuclear antigen; NSCLC: non-small lung cancer; TCGA: The Cancer Genome Atlas; GEO: Gene Expression Omnibus; IHC: immunochemistry; qRT-PCR: quantitative real-time PCR; IF: immunofluorescence; OS: overall survival; HR: hazard ratio; 95\% CI: 95\% confidence interval; ROC: receiver operating characteristic; AUC: area under the curve; HCC: hepatocellular carcinoma; cDNA: complementary DNA; FFPE: formalin-fixed paraffin-embedded; OD: optical density; GSEA: gene set enrichment analysis; NES: normalized enrichment score; FDR: false discovery rate; CTLA-4: cytotoxic T-lymphocyte-associated antigen 4; PD-1: programmed death 1; OEATC-1: overexpressed in anaplastic thyroid carcinoma-1; AFP: alpha-fetoprotein; CEA: carcinoembryonic antigen; EMT: epithelial-to-mesenchymal transition; TNM stage: tumor-node-metastasis stage.

\section{Acknowledgments}

This work was supported by grants from the National Natural Science Foundation of China (Grant No: 81972179 to Jianya Zhou).

\section{Competing Interests}

The authors have declared that no competing interest exists.

\section{References}

1. Siegel RL, Miller KD, Jemal A. Cancer statistics, 2019. CA Cancer J Clin. 2019; 69: 7-34.

2. Chang CN, Feng MJ, Chen $\mathrm{YL}$, et al. p15(PAF) is an Rb/E2F-regulated S-phase protein essential for DNA synthesis and cell cycle progression. PLoS One. 2013; 8: e61196.

3. Kais Z, Barsky SH, Mathsyaraja H, et al. KIAA0101 interacts with BRCA1 and regulates centrosome number. Mol Cancer Res. 2011; 9: 1091-9.

4. Emanuele MJ, Ciccia A, Elia AE, et al. Proliferating cell nuclear antigen (PCNA)-associated KIAA0101/PAF15 protein is a cell cycle-regulated anaphase-promoting complex/cyclosome substrate. Proc Natl Acad Sci U S A. 2011; 108: 9845-50.

5. Lv W, Su B, Li Y, et al. KIAA0101 inhibition suppresses cell proliferation and cell cycle progression by promoting the interaction between p53 and Sp1 in breast cancer. Biochem Biophys Res Commun. 2018; 503: 600-6.

6. Jain N, Roy J, Das B, et al. miR-197-5p inhibits sarcomagenesis and induces cellular senescence via repression of KIAA0101. Mol Carcinog. 2019; 58: 1376-88

7. Guo M, Li J, Wan D, et al. KIAA0101 (OEACT-1), an expressionally down-regulated and growth-inhibitory gene in human hepatocellular carcinoma. BMC Cancer. 2006; 6: 109

8. Kato T, Daigo Y, Aragaki M, et al. Overexpression of KIAA0101 predicts poor prognosis in primary lung cancer patients. Lung Cancer. 2012; 75: 110-8. 
9. Martinez P, Peters S, Stammers T, et al. Immunotherapy for the First-Line Treatment of Patients with Metastatic Non-Small Cell Lung Cancer. Clin Cancer Res. 2019; 25: 2691-8.

10. Mailand N, Gibbs-Seymour I, Bekker-Jensen S. Regulation of PCNA-protein interactions for genome stability. Nat Rev Mol Cell Bio. 2013; 14: 269-82.

11. van Bueren KL, Bennetts JS, Fowles LF, et al. Murine embryonic expression of the gene for the UV-responsive protein p15(PAF). Gene Expr Patterns. 2007; 7: 47-50.

12. Jain M, Zhang L, Patterson EE, et al. KIAA0101 is overexpressed, and promotes growth and invasion in adrenal cancer. PLoS One. 2011; 6: e26866.

13. Jin C, Liu Z, Li Y, et al. PCNA-associated factor P15(PAF), targeted by FOXM1, predicts poor prognosis in high-grade serous ovarian cancer patients. Int J Cancer. 2018; 143: 2973-84.

14. Zhu K, Diao D, Dang C, et al. Elevated KIAA0101 expression is a marker of recurrence in human gastric cancer. Cancer Sci. 2013; 104: 353-9.

15. Abdelgawad IA, Radwan NH, Hassanein HR. KIAA0101 mRNA expression in the peripheral blood of hepatocellular carcinoma patients: Association with some clinicopathological features. Clin Biochem. 2016; 49: 787-91.

16. Zhang T, Guo J, Gu J, et al. KIAA0101 is a novel transcriptional target of FoxM1 and is involved in the regulation of hepatocellular carcinoma microvascular invasion by regulating epithelial-mesenchymal transition. J Cancer. 2019; 10: 3501-16.

17. Bouska A, Eischen CM. Mdm2 affects genome stability independent of p53. Cancer Res. 2009; 69: 1697-701.

18. Laptenko $\mathrm{O}$, Prives $\mathrm{C}$. Transcriptional regulation by $\mathrm{p} 53$ : one protein, many possibilities. Cell Death Differ. 2006; 13: 951-61.

19. Cheng YW, Liao LD, Yang Q, et al. The histone deacetylase inhibitor panobinostat exerts anticancer effects on esophageal squamous cell carcinoma cells by inducing cell cycle arrest. Cell Biochem Funct. 2018; 36: 398-407. 